\title{
Surface properties of a single perfluoroalkyl group on water surfaces studied by surface potential measurements
}

\section{$\operatorname{AUTHOR}(\mathrm{S})$ :}

Shimoaka, Takafumi; Tanaka, Yuki; Shioya, Nobutaka; Morita, Kohei; Sonoyama, Masashi; Amii, Hideki; Takagi, Toshiyuki; Kanamori, Toshiyuki; Hasegawa, Takeshi

\section{CITATION:}

Shimoaka, Takafumi ...[et al]. Surface properties of a single perfluoroalkyl group on water surfaces studied by surface potential measurements. Journal of Colloid and Interface Science 2016, 483: 353-359

\section{ISSUE DATE:}

2016-12-01

URL:

http://hdl.handle.net/2433/216978

\section{RIGHT:}

(c) 2016. This manuscript version is made available under the CC-BY-NC-ND 4.0 license

http://creativecommons.org/licenses/by-nc-nd/4.0/; The full-text file will be made open to the public on 1 December 2018 in accordance with publisher's 'Terms and Conditions for Self-Archiving'.; この論文は出版社版でありません。引 用の際には出版社版をご確認ご利用ください。; This is not the published version. Please cite only the published version. 


\section{Surface Properties of a Single Perfluoroalkyl Group} on Water Surfaces Studied by Surface Potential

$$
\text { Takafumi Shimoaka }{ }^{a} \text {, Yuki Tanaka }{ }^{a}, \text { Nobutaka Shioya }^{a}, \text { Kohei Morita }^{b} \text {, }
$$
Masashi Sonoyama ${ }^{b}$, Hideki Amii ${ }^{b}$, Toshiyuki Takagi ${ }^{c}$, Toshiyuki Kanamori ${ }^{c}$,

${ }^{a}$ Laboratory of Solution and Interface Chemistry, Division of Environmental Chemistry, Institute for Chemical Research, Kyoto University, Gokasho, Uji, Kyoto 611-0011, Japan

${ }^{\mathrm{b}}$ Division of Molecular Science, Faculty of Science and Technology, Gunma University, Tsukuba Central 5, 1-1-1 Higashi, Tsukuba, Ibaraki 305-8565, Japan

E-mail address: [TS] shimoaka@,scl.kyoto-u.ac.jp [YT] yuki@env.kuicr.kyoto-u.ac.jp

[NS] nobutaka@env.kuicr.kyoto-u.ac.jp [KM] t09301167@gunma-u.ac.jp 
*Corresponding Author: Prof. Takeshi Hasegawa

2 Postal address: Laboratory of Solution and Interface Chemistry, Division of

5

6

7

8

9

10 Highlights:

- Dehydration about the head group is a key to understand surface potential changes.

- A single perfluoroalkyl group is stabilized on a water surface.

- A long perfluoroalkyl chain makes a stiff molecular domain on the water surface.
Environmental Chemistry, Institute for Chemical Research, Kyoto

University, Gokasho, Uji, Kyoto 611-0011, Japan

Phone: $\quad+81774383070$

FAX: $\quad+81774383074$

E-mail: $\quad$ htakeshi@scl.kyoto-u.ac.jp 
1 Abstract: A discriminative study of a single perfluoroalkyl (Rf) group from a bulk

2 material is recently recognized to be necessary toward the total understanding of Rf

3 compounds based on a primary chemical structure. The single molecule and the bulk

4 matter have an interrelationship via an intrinsic two-dimensional (2D) aggregation

5 property of an Rf group, which is theorized by the stratified dipole-arrays (SDA) theory.

6 Since an $\mathrm{Rf}$ group has dipole moments along many $\mathrm{C}-\mathrm{F}$ bonds, a single $\mathrm{Rf}$ group would

7 possess a hydrophilic-like character on the surface. To reveal the hydration character of

8 a single $\mathrm{Rf}$ group, in the present study, surface potential $(\Delta V)$ measurements are

9 performed for Langmuir monolayers of Rf-containing compounds. From a comparative

10 study with a monolayer of a normal hydrocarbon compound, the hydration/dehydration

11 dynamics of a lying $\mathrm{Rf}$ group on water has first been monitored by $\Delta V$ measurements,

12 through which a single Rf group has been revealed to have a unique "dipole-interactive"

13 character, which enables the Rf group interacted with the water 'surface.' In addition,

14 the SDA theory proves to be useful to predict the $2 \mathrm{D}$ aggregation property across the

15 phase transition temperature of $19^{\circ} \mathrm{C}$ by use of the $\Delta V$ measurements.

16 Keywords: Langmuir monolayer; surface potential; single perfluoroalkyl chain; water

17 surface 
1 Introduction: Perfluoroalkyl (Rf) compounds exhibit unique "bulk characters"

2 represented by the high water- and oil-repelling property, low dielectric permittivity, and

3 low solubility in a solvent, which have long been used extensively for many practical

4 chemical products. ${ }^{1}$ When a local structure of the $\mathrm{Rf}$ group is taken into account,

5 however, uniformed understanding of the bulk properties is difficult. For example, the

$6 \mathrm{C}-\mathrm{F}$ bond has a large dipole moment due to the largest electronegative character of

7 fluorine, ${ }^{2,3}$ which should attract water molecule via the dipole-dipole interaction. This

8 fact seems inconsistent with the hydrophobic surface property on $\mathrm{Rf}$ compounds.

9 Recently, a novel chemical theory, i.e., the stratified dipole-arrays (SDA) theory, has

10 been proposed for fully understanding the material characters, which discriminates a

11 'single molecular character' from the 'bulk one. ${ }^{4}$

This theory is based on the dipole-dipole interaction ${ }^{5}$ of the $\mathrm{Rf}$ groups having a

13 helical structure. ${ }^{1,6,7}$ In $\mathrm{Rf}$ compounds, the $\mathrm{Rf}$ chains are aggregated tightly by

14 two-dimensional dipole-dipole interaction arrays, in which all the dipoles corresponding

15 to $\mathrm{CF}_{2}$ groups are linearly aligned in the head-to-tail manner. The tight aggregation

16 readily explains most of the bulk characters comprehensively ${ }^{4}$. Fortunately, this theory

17 readily covers the conventional polarizability-based theory ${ }^{8}$ to account for the low

18 permittivity. Since the intrinsic difference between an $\mathrm{Rf}$ group and a normal 
1 hydrocarbon is attributed ${ }^{9}$ to the difference between the dipole-dipole interaction and

2 the dispersion force, ${ }^{5}$ respectively, the SDA theory built on the dipole-dipole interaction

3 has become very important. In particular, the SDA theory is the only theory accounting

4 for the discontinuity of the melting point ${ }^{4}$ at the length of $-\mathrm{C}_{8} \mathrm{~F}_{17}$ as well as the

5 fluorophilic effect ${ }^{10}$ and the low electric permittivity, which are not found in a normal

6 hydrocarbon material.

Some of the 'single molecular characters' predicted by the SDA theory have experimentally been confirmed via the adsorption of 'molecular water' on a stretched polytetrafluoroethylene (PTFE; known as Teflon ${ }^{\circledR}$ ) tape by using ${ }^{1} \mathrm{H}^{\mathrm{NMR}}{ }^{11}$. A PTFE tape is made of bundled fibrils of polymeric Rf chains with the SDA packing. If the

11 tape is mechanically stretched, then the SDA packed fibrils are partly disaggregated, and a single-molecule character of an Rf chain faces to the air. Since a single Rf group possesses strong dipole moments on the surface due to the $\mathrm{C}-\mathrm{F}$ bonds, molecular water

14 having a large dipole should be adsorbed on the surface via the dipole-dipole interaction. The ${ }^{1} \mathrm{H}$ NMR study reveals that the adsorption is due to the dipole-diploe interaction in fact by the chemical shift and $T_{1}$ analyses. 
1 the polarization density (summation of dipole moment in a macroscopic scale)

2 becomes nearly zero for both cases. In fact, a large contact angle is commonly obtained

3 for both cases ${ }^{11}$.

This implies that the attraction character of a single $\mathrm{Rf}$ group to a molecular

5 study of the LB film. ${ }^{4}$ For example, according to the theory, a compound containing a water should be discriminated from the general concept of hydrophilicity, and the character is thus called "dipole-interactive property" throughout this paper. In the present study, the physical character of a single $\mathrm{Rf}$ group is investigated at the air/water interface. Water surface is known to have some hydrogen-bond free (dangling) $\mathrm{OH}$ groups, ${ }^{12}$ which should be a good field to study a single Rf group on the concept of the dipole-interactive property. A study using a spread monolayer on water (Langmuir (L) film) and the Langmuir-Blodgett (LB) film is a key approach to investigate properties of a two-dimensional (2D) molecular aggregate. ${ }^{13}$ In fact, the SDA theory has already proved to be powerful to predict the material property of a compound by the surface pressure $(\pi)$-surface area $(A)$ isotherm measurement of the $\mathrm{L}$ film and a spectroscopic study of the LB film. For example, according to the theory, a compound containing a short Rf-group $\left(\mathrm{CF}_{3}\left(\mathrm{CF}_{2}\right)_{6}-\right.$ or shorter $)$ should exhibit the dipole interactive or hydrophilic character, especially when the compound is spread on water: the spread 
1 molecules are not aggregated, and they are lying on the water surface. ${ }^{4}$ In fact, the

2 monolayer stays on pure water stably, and the lift-off surface area is apparently larger

3 than that of a similar compound having no Rf group. ${ }^{13}$ This implies that the short-Rf

4 containing compounds are not dissolving into water, but it is strongly interacted with the

5 water 'surface.' In this manner, a single $\mathrm{Rf}$ group should have a unique attracting

6 character with the water surface, which is not found for a normal hydrocarbon.

7 To reveal the unique property of an $\mathrm{Rf}$ group on a water surface, in the present

8 study, the surface potential $(\Delta V)$-surface area $(A)$ isotherms are measured for some

9 Rf-containing myristic acid (MA) derivatives having an Rf group with a different length,

10 which are cooperatively discussed with the $\pi-A$ isotherms. As a result, a notable

11 molecular aggregation property depending on both $\mathrm{Rf}$ length and temperature is

12 revealed. Thus far, $\Delta V-A$ isotherms have been discussed in terms of molecular

13 orientation change after Gains's textbook. ${ }^{15}$ In the present study, however, additional

14 two factors are found necessary: the molecular density change and the dehydration

15 about the head group on the monolayer compression. The dehydration is particularly

16 useful to discuss the dipole-interactive property on the water surface, since the

17 dipole-shielding by the hydration water is broken by the monolayer compression.

In addition, the $\Delta V-A$ isotherms reveal that bulk properties on the phase 
1 transition can also be predicted by the SDA theory. The measurements have all been

2 performed thus far at $15{ }^{\circ} \mathrm{C}$ (Phase II) below the transition temperature at $19{ }^{\circ} \mathrm{C} .{ }^{16}$ If the

3 Rf compounds obey the phase diagram, ${ }^{16}$ the SDA theory should readily predict the

4 bulk properties at another temperature above $19{ }^{\circ} \mathrm{C}$ (i.e., Phase $\mathrm{IV}^{16}$ ). In the present

5 study, the same analysis is also performed at $25^{\circ} \mathrm{C}$ in Phase IV, and the SDA theory has

6 proved to be powerful for explaining the phase transition. This is the first report to our

7 knowledge that the bulk characters of Rf-containing compounds are predicted based on

8 the primary chemical structure.

10 Materials and methods:

11 Sample preparation: Octadecanoic acid (stearic acid, SA, $\geq 98.5 \%$ ), tetradecanoic

12 acid (myristic acid, MA, $\geq 99 \%$ ) and chloroform (ACS Spectra Grade, $\geq 99.8 \%$ ) were

13 purchased from Sigma-Aldrich (St. Louis, MO, USA), and they were used as is without

14 further purification. 11,11,12,12,13,13,14,14,14-Nonafluorotetradecanoic acid (MA-

15 Rf3), 9,9,10,10,11,11,12,12,13,13,14,14,14-tridecafluorotetradecanoic acid (MA-Rf5),

$167,7,8,8,9,9,10,10,11,11,12,12,13,13,14,14,14$-heptadecafluorotetradecanoic acid (MA-

17 Rf7) and 5,5,6,6,7,7,8,8,9,9,10,10,11,11,12,12,13,13,14,14,14-henicosafluorotetra-

18 decanoic acid (MA-Rf9) were synthesized as mentioned in our previous work. ${ }^{4}$ Each 
1 amphiphilic compound was dissolved in chloroform with a concentration of $0.2-1.0 \mathrm{mg}$

$2 \mathrm{~mL}^{-1}$ for preparation of a Langmuir monolayer on water.

$3 \boldsymbol{\pi}-\boldsymbol{A}$ and $\Delta \boldsymbol{V}-\boldsymbol{A}$ measurements: The $\pi-A$ and $\Delta V-A$ isotherms were simultaneously

4 measured by using a Biolin Scientific (Espoo, Finland) KSV-NIMA Minitrough

5 Langmuir-Blodgett system equipped with a KSV-NIMA SPOT Kelvin probe. The $\pi$ was

6 measured by the Wilhelmy method using a paper plate with a width of $1 \mathrm{~cm}$, and $\Delta V$

7 was measured by using the Kelvin probe with a counter electrode made of stainless

8 steel. ${ }^{17}$ The Kelvin probe (KP) was located at the position (1) in Chart 1. Each isotherm

9 was measured three times to check the reproducibility. The water subphase and room

10 temperature were both maintained at $15^{\circ} \mathrm{C}$ or $25^{\circ} \mathrm{C}$ to keep the phase II $\left(<19{ }^{\circ} \mathrm{C}\right)$ or IV

$11\left(>19{ }^{\circ} \mathrm{C}\right)$, respectively. Pure water subphase was obtained by a Millipore (Molsheim,

12 France) Elix UV-3 pure-water generator and a Yamato (Tokyo, Japan) Autopure

13 WT100U water purifier (a compatible model with Milli-Q). The water exhibited an

14 electric resistivity of $18.2 \mathrm{M} \Omega \mathrm{cm}$ or higher. The surface tension was $72.5 \mathrm{mN} \mathrm{m}^{-1}$ at

$1525^{\circ} \mathrm{C}$, which was measured by using a Kyowa Interface Science Co., Ltd. (Saitama,

16 Japan) DropMaster, DM-501Hy, contact angle meter. The Langmuir monolayer was

17 prepared by spreading the chloroform solution of the amphiphilic compounds on pure

18 water subphase with a size of $75 \times 324 \mathrm{~mm}^{2}$. Each isotherm was measured with a 
1 compression rate of $1.688 \times 10^{3} \mathrm{~mm}^{2} \mathrm{~min}^{-1}$.

2

3

4

5

6

7

8

9

The isotherms were measured on pure water (ca. $\mathrm{pH} 5.8$ ) to make the potential measurements free from a chemical interference due to a $\mathrm{pH}$ modifier. For only MA, the isotherms were measured at $\mathrm{pH} 2.0$; otherwise the shorter compound than $\mathrm{SA}$ is dissolved into the subphase. ${ }^{15}$ The isotherms of a MA monolayer were measured on a subphase of $\mathrm{pH} 2.0$ involving hydrochloride to prevent the dissolution of the monolayer into the subphase. ${ }^{12}$

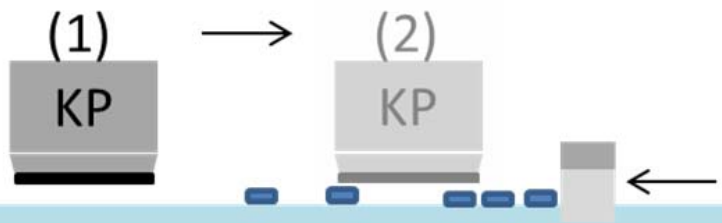

Chart 1 The relative position of the Kelvin probe to the trough. The dark blue objects are island domains of a Langmuir film. The surface potential $(\Delta V)$ of the film is measured at the position (1) except for the purple dash line in Figure $3 \mathrm{a}$, which is measured at the position (2).

6 Results and Discussion: The variation of $\Delta V$ during the monolayer compression $(\Delta V-$

17 A) is discussed with related to the corresponding $\pi-A$ isotherm. A $\Delta V-A$ isotherm of a

Langmuir monolayer ( $\mathrm{L}$ film) is often discussed in terms of only the molecular orientation change of a chemical group having a large dipole moment. ${ }^{18}$ In the following 
1 sections, we show that a $\Delta V-A$ isotherm reflects two other factors, molecular density

2 and dehydration about a polar head group, as well as the orientation change, which has

3 an apparent correlation with the $\pi-A$ isotherm. Before discussing the Rf-containing

4 compounds, L films of commonly known stearic acid (SA) and MA are thus used to

5 show the newly found key factors.

$6 \Delta V-A$ isotherm of a Normal Carboxylic Acid:

7

Figure 1a presents $\pi-A$ and $\Delta V-A$ isotherms of an $\mathrm{L}$ film of SA measured at

$8 \quad 25^{\circ} \mathrm{C}$. The $\pi-A$ isotherm has a typical shape consisting of the well-known three regions:

9 the gaseous and liquid condensed (G/LC), LC (or "tilted condensed") and solid (S, or

10 "un-tilted condensed") phase regions. ${ }^{19,20}$ The electric dipole moment of SA is localized

11 near the $\mathrm{C}=\mathrm{O}$ group, but the moment $(1.50 \mathrm{D})^{21}$ is tilted to have the upward component

12 (Figure 1c). Thus, the dipole moment can readily be monitored by the Kelvin probe

13 even when the molecule has a perpendicular stance to the water surface. ${ }^{15}$ In fact, $\Delta V$

14 positively appears in the $\mathrm{S}$ phase. Note that the $\Delta V$ - $A$ isotherm seems not synchronous to

15 the $\pi-A$ curve (Figure 1a). In particular, $\Delta V$ begins to increase at ca. $A=0.35 \mathrm{~nm}^{2}$

16 molecule $\mathrm{e}^{-1}$, which is apparently larger than the lift-off of $\pi\left(\right.$ ca. $0.25 \mathrm{~nm}^{2}$ molecule $\left.{ }^{-1}\right)$ in

17 the $\mathrm{G} / \mathrm{LC}$ region. 


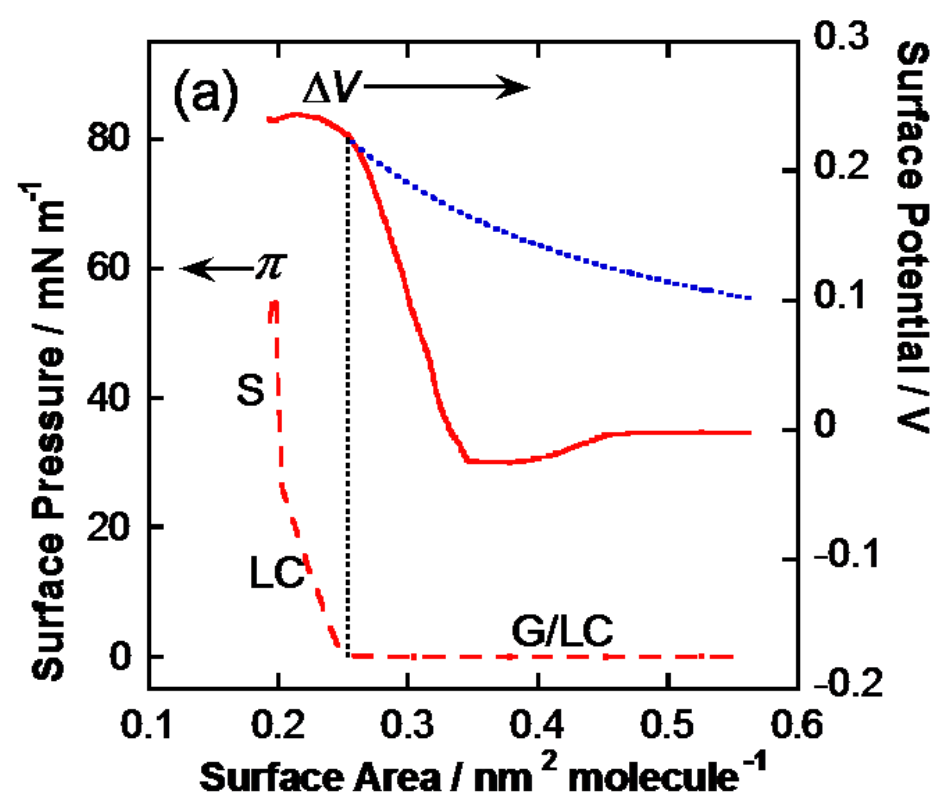

(c)
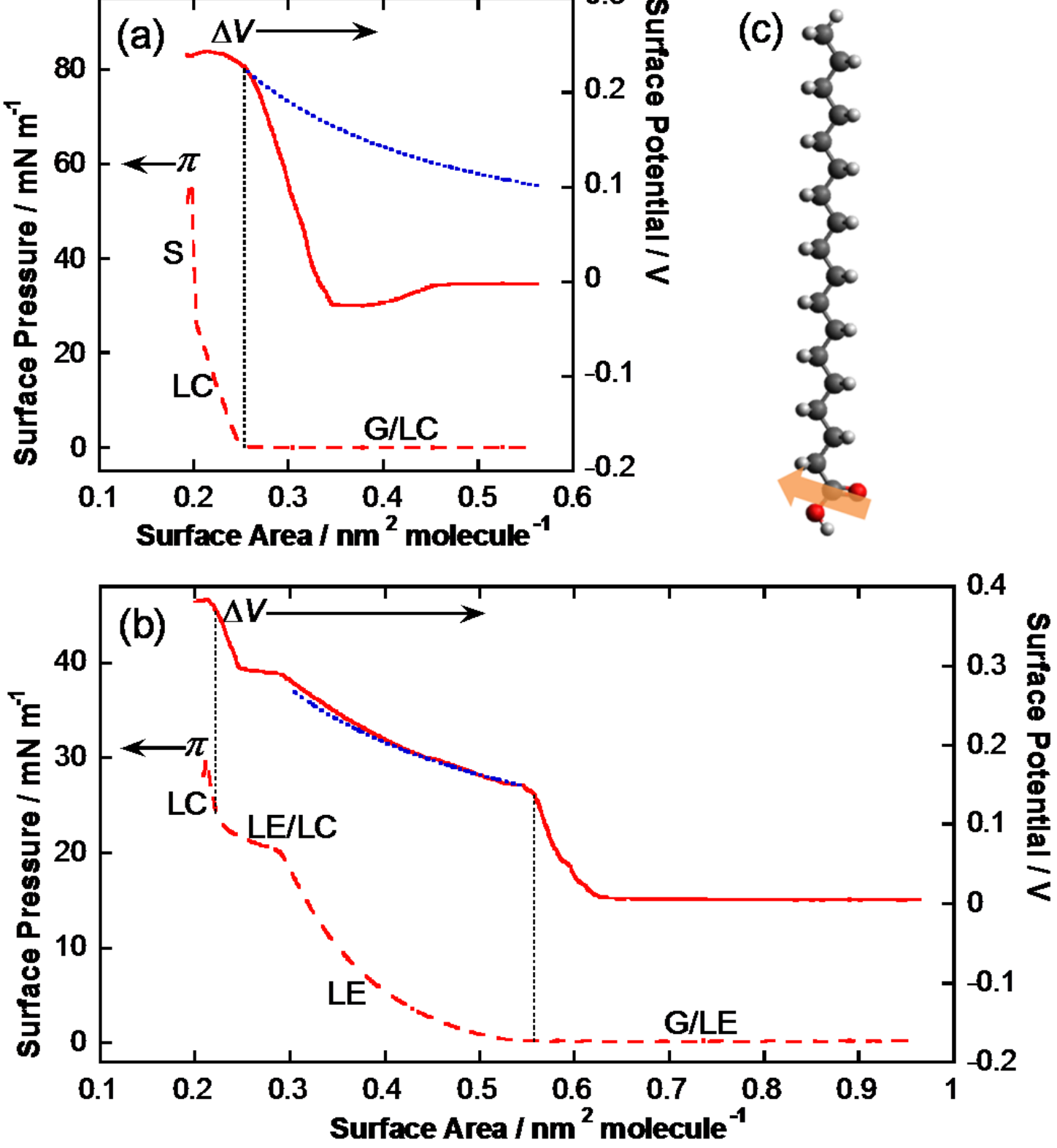

2 Figure 1. The $\pi-A$ (dashed red line) and $\Delta V-A$ (solid red line) isotherms of a Langmuir monolayer of (a) stearic acid (SA) and (b) myristic acid (MA) measured at $25{ }^{\circ} \mathrm{C}$. The blue dotted line in the panel (a) and (b) is a calculated "density curve" (see the text for detail). (c) A molecular schematic of stearic acid. The orange arrow represents the molecular dipole moment calculated by a quantum chemical calculation. 
In this region, the monolayer compression corresponds to the collection of the

2 island domains as confirmed by the Brewster angle microscopic study, ${ }^{22}$ which accounts

3 for no increase of the surface pressure $(\pi \approx 0)$. The domain is actually an aggregate of

4 loosely packed SA molecules, but the aggregation property is apparently larger than MA

5 because of the difference of the chain length. ${ }^{23}$

Once $\Delta V$ begins to increase, the slope should be influenced by changes of the molecular density and the orientation. Before attaining the LC region (i.e., in the G/LC region), fortunately, the orientation change can be ignored. To consider the density

9 change, a theoretical "density curve" is calculated by Eq. 1.

$$
\Delta V_{\text {density }}=\Delta V_{0} A_{0} / A
$$

$11 A$ is the surface area and $A_{0}$ is the end area of the $\mathrm{G} / \mathrm{LC}$ region exhibiting $V_{0}$. The point,

$12\left(A_{0}, \Delta V_{0}\right)$ is read from the isotherm to be $(0.254,0.225)$, and the calculated density

13 curve is presented by the blue dotted curve in Figure 1a. As a result, the density curve

14 cannot quantitatively explain the $\Delta V-A$ curve at all, which straightforwardly implies

15 that the third factor should be considered. To explain the sudden appearance of $\Delta V$ at

16 about $0.35 \mathrm{~nm}^{2}$ molecule ${ }^{-1}$, not only the hydrocarbon chain of SA, but also the head group should also be taken into account. 
1 shielded by the dipoles of surrounding water molecules. This explains that $\Delta V$ exhibits

2 nearly zero at a surface area larger than ca. $0.35 \mathrm{~nm}^{2}$ molecule ${ }^{-1}$. On a further

3 compression, $\Delta V$ runs up to $0.23 \mathrm{~V}$ before $\pi$ increases. This sudden appearance of $\Delta V$ is

4 understandable by considering the removal of the hydration water by the monolayer

5 compression. In other words, the dehydration about the carboxylic group is induced by

6 the monolayer compression, after which the molecules are aggregated resulting in the

7 increase of $\pi$. Therefore, the lift-off of $\pi$ is always found at the surface area where the

8 increase of $\Delta V$ finishes. In the $\mathrm{S}$ region, no potential change is monitored, which is

9 reasonable when considering that almost no change in the density, orientation and

10 dehydration is expected in the very little change of the surface area.

In this manner, by considering the dehydration process, the variations of $\pi$ and

$12 \Delta V$ on the compression are comprehensively explained. The relative position of the

13 increase of $\pi$ and $\Delta V$ is thus found very useful to discuss the dehydration; whereas the

14 absolute value of $\Delta V$ is not so important.

On this fundamental mechanism, the isotherms of an L film of MA are next

discussed. When a monolayer of MA is compressed on pure water ( $\mathrm{pH} 5.8$ ), the $\pi-A$ isotherm shifts to a smaller area due to the dissolution of the molecules into the subphase because the carboxylic group of MA are partially ionized ${ }^{24}$. To prevent the 
matter, the isotherms of only MA were measured at $\mathrm{pH} 2.0$. A $\pi-A$ isotherm of the MA

2 at $\mathrm{pH} 2.0$ is a little bit shifted to a larger area than that on pure water. Fortunately,

3 however, $\Delta V-A$ curve also exhibits the same shift. Therefore, the discussion of the

4 relative position between the $\pi-A$ and $\Delta V-A$ isotherms is impervious to $\mathrm{pH}$. monolayer involves the LE and LE/LC phases in addition to the SA isotherm. This is

7 because the molecular aggregation property of MA, having shorter alkyl chain, is 8 weaker than that of SA. ${ }^{19}$

In the case of MA, $\Delta V$ keeps nearly zero until ca. $0.63 \mathrm{~nm}^{2}$ molecule ${ }^{-1}$ and suddenly increases up to $0.15 \mathrm{~V}$ before $\pi$ increases (Figure 1c). This can be explained by the dehydration model as found for SA. In the LE region, $\Delta V$ increases almost along the density curve (blue dotted curve in Figure 1c, calculated by Eq. 1 with $\left(A_{0}, \Delta V_{0}\right)=$ $(0.544,0.150))$, which suggests that the potential change can simply be attributed to the increase of molecular density only. The LE/LC region is known to correspond to the molecular orientation change

16 during the film compression. ${ }^{19} \Delta V$ in the LE/LC region keeps almost a constant value.

17 This means that a decrease of $\Delta V$ due to the orientation change cancels the density contribution. The decrease is attributed to the orientation change of MA having the 
1 carboxylic group. 6 during the compression.

At the near end of the LE/LC region, $\Delta V$ rapidly increases again. In the LC regions, the trends of the $\Delta V-A$ and $\pi-A$ isotherms are almost the same as those of SA. Therefore, the rapid increase is again attributed to the dehydration of the carboxylic group. As a conclusion, the dehydration of MA is suggested to occur twice stepwisely

7 Analysis of MA-Rfn monolayers via Surface Potential Isotherms at $15{ }^{\circ} \mathrm{C}$ (Phase

8 II): The $\pi-A$ isotherms of MA-Rf7 and MA-Rf9 (Figure 2a) in the present study are 9 identical to those in the previous papers, including partially fluorinated alkanes, 10 alcohols, ${ }^{4,25-29}$ in which no gas and LE region appear in the curves followed by a steep 11 increase of $\pi$. This apparently implies that the MA-Rf7 and MA-Rf9 commonly have a spontaneous aggregation character. ${ }^{4}$ To discuss the $\Delta V-A$ isotherms of MA-Rfn (Figure 2a and 3), the molecular dipole moment of the compounds should be noted. Whereas the molecular dipole moment of SA and MA is nearly localized at the $\mathrm{C}=\mathrm{O}$ group, MA-Rfn has a large dipole moment along the $R f-R$ bond represented as the large arrow in Figure

$162 \mathrm{~b}$ and the contribution of $\mathrm{C}=\mathrm{O}$ is minor as a whole. ${ }^{1}$ Since the molecules of MA-Rf7 and MA-Rf9 in a monolayer at $15{ }^{\circ} \mathrm{C}$ (Phase II) are oriented perpendicular to the water

18 surface, ${ }^{4,28}$ the dipole moment with a 'downward' direction should have a large 
1 surface-normal component to yield a large 'negative' $\Delta V$.

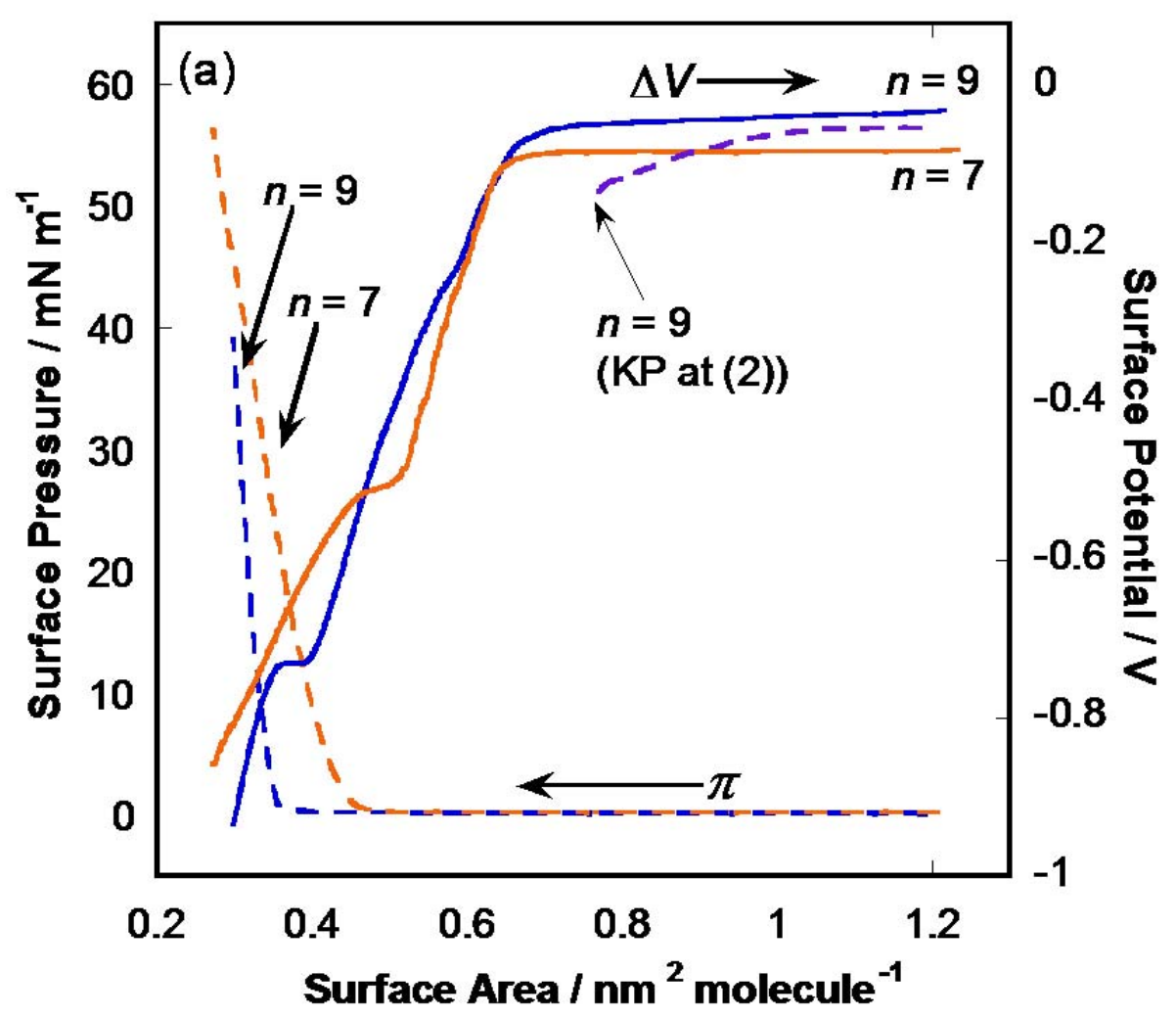

(b)

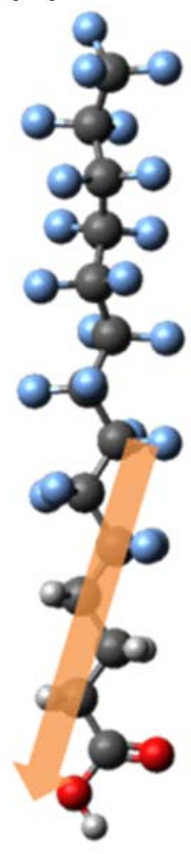

Figure 2. (a) The $\pi-A$ (dashed line) and $\Delta V-A$ (solid line) isotherms of Langmuir monolayers of MA-Rf7 (orange) and MA-Rf9 (blue) at $15{ }^{\circ} \mathrm{C}$ (phase II). The $\Delta V-A$ isotherm of MA-Rf9 displayed in purple is measured with the $\mathrm{KP}$ at the position of (2) in Chart 1. (b) A molecular schematic of MA-Rf9. The orange arrow represents the molecular dipole moment. The blue atoms are fluorines.

In fact, as the monolayer is compressed, $\Delta V$ changes to a very large negative value (Figure $2 \mathrm{a}$ ), which is specific to a diblock ( $\mathrm{Rf}-\mathrm{R}$ ) compound. ${ }^{27,}{ }^{30} \Delta V$ is constant at nearly zero at a surface area larger than ca. $0.70 \mathrm{~nm}^{2}$ molecule ${ }^{-1}$. In the case of SA and 
1 MA, the constant potential at nearly zero was attributed to the hydration of the

2 carboxylic group. As for MA-Rf9, a more primitive phenomenon readily explains it,

3 that is, the inhomogeneous distribution of the island domains. According to the SDA

4 theory, the MA-Rf9 molecules aggregate spontaneously, and a stiff island domain are

5 generated. ${ }^{4}$ When a monolayer of MA-Rf9 is compressed by the moving barrier, the

6 stiff domains should be accumulated near the barrier; whereas the domains near the

7 Kelvin probe at the position (1) in Chart 1 would be left un-compressed at the initial 8 stage.

To confirm this speculation, the same measurements were carried out by

10 displacing the Kelvin probe to the position (2) in Chart 1. As expected, $\Delta V$ decreases

11 earlier (the purple dashed line in Figure 2a), which proves that the stiff domains near the

12 barriers are collected to arrive at the position (2) earlier. The inhomogeneity model has

13 thus been confirmed readily. MA-Rf7 (orange curve) exhibits a similar process, but the

14 initial constant potential is a little shifted to a negative value, which will be discussed in

15 detail in the next section.

During the decrease of $\Delta V$, an "apparent plateau" appears just before the lift-off

17 of $\pi$ for both MA-Rf7 $(A \approx 0.5)$ and MA-Rf9 $(A \approx 0.4)$. The relative position of the 
1 should be attributed to the dehydration of the carboxylic group, since the dehydration

2 generates the positive increase of the potential.

In the case of SA and MA, the potential increase due to the dehydration cannot

4 be discriminated from the increasing density, since both changes exhibits positive

5 changes. On the other hand, in the case of an Rf-R diblock compound, the two changes

6 exhibit opposite potential changes, which make the dehydration process clearly visible. chain have a poor aggregation property, which is quite different from MA-Rf7 and

9 MA-Rf9. ${ }^{4}$ As found in the isotherms in Figure 3, $\pi$ exhibits an apparent positive value 10 even at an initial compression, which indicates that molecules are lying on the water

11 surface. ${ }^{4}$ As discussed for normal MA shown in Figure 1b, the spread molecules do not make island domains, but they are spread over the water surface homogeneously. the $\pi-A$ isotherms in Figure 3. The $\Delta V$ curves of MA-Rf3 and MA-Rf5 decrease significantly as compressed, which agrees with the density curve (blue dotted curve; $\left(A_{0}\right.$,

$\left.\left.16 \Delta V_{0}\right)=(1.211,-0.208)\right)$. This agreement confirms that the molecules are, in fact, homogeneously spread over the water surface for both compounds. 

9 negative value considering various molecular rotational orientations of the lying

10 molecule (Figure 4a-c). Water surface is known to have hydrogen-bond free (dangling)

$11 \mathrm{OH}$ groups, ${ }^{12}$ which can receive a single Rf group via the dipole-dipole interaction. In

Figure 3. The $\pi-A$ (dashed line) and $\Delta V-A$ (solid line) isotherms of Langmuir monolayers of MA-Rf3 (red) and MA-Rf5 (green) at $15{ }^{\circ} \mathrm{C}$ (phase II). The blue dotted line is a calculated density curve.

Here, we have to pay attention to a fact that $\Delta V$ exhibits a fairly large negative value "even before" the monolayer compression, which is apparently larger than that in Figure $2 \mathrm{a}$. If the molecules are lying on the water surface, there is no need to exhibit a

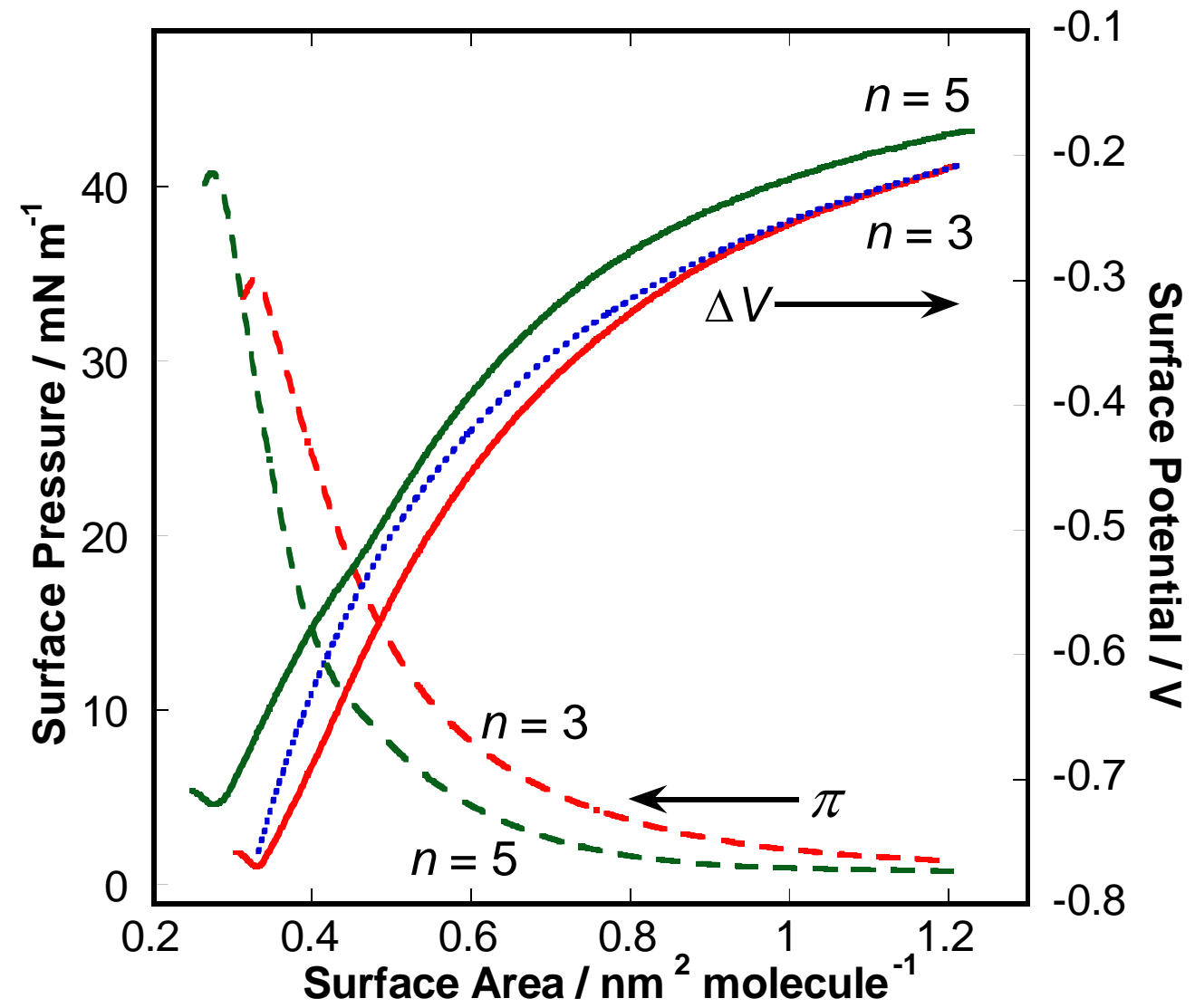


1 this manner, a single Rf group lying on the water surface would be highly stabilized via

2 the dipole-interactive property. In addition, a single $\mathrm{Rf}$ group is not dissolved into a

3 bulky water, since the Rf group has only the dipole-interactive character, and no

4 hydrogen bonding one, which is unfavorable to break the hydrogen bonding network of

5 the bulky water. As a result, a single Rf group is stably interacted with water 'surface'

6 only.

7

8

9

10

11

12 (a)

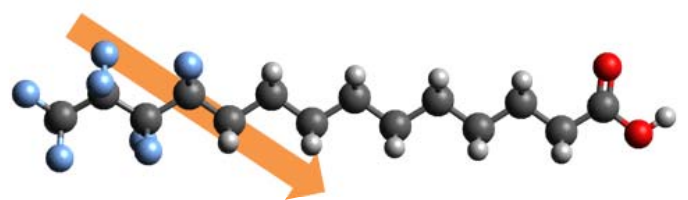

(b)

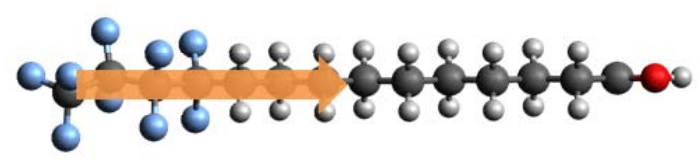

(c)

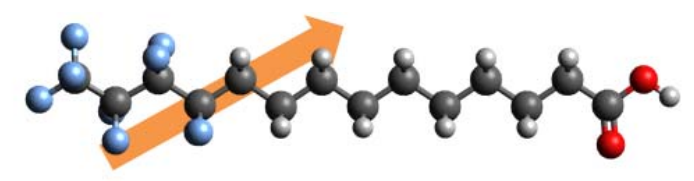

(d)

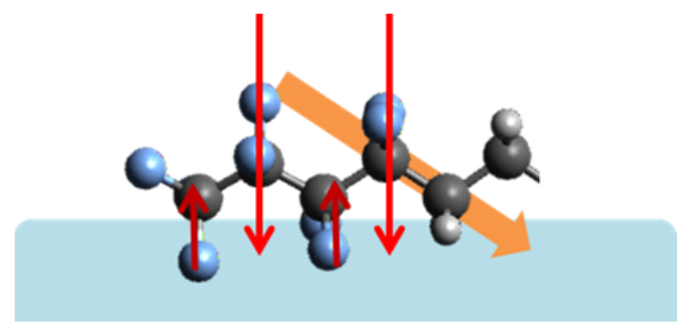

Figure 4. (a) (c) Three different rotational orientations of a lying MA-Rf3 molecule. (d) A schematic view of a dipole-interactive $\mathrm{Rf}$ group in the water surface based on the schematic (a). 
2 isotherms. In this model, one side of the lying Rf group is attached to the water surface,

3 and the rest half remains un-hydrated. This partial hydration model implies that each

4 dipole moment on the hydrated side is shielded, and the negative potential should

5 appear as a total irrespective of the rotational molecular orientation on the water surface.

7 Prediction of Phase Transition by the SDA theory: The analytical discussion made

8 above is for a fixed temperature of $15^{\circ} \mathrm{C}$, i.e., Phase II. In the present section, the bulk

9 material properties of monolayers in Phase IV $^{12}$ are predicted on the SDA theory.

The SDA theory provides a criterion ${ }^{4}$ whether the spontaneous two-dimensional

11 molecular aggregation of Rf groups takes place or not, which depends on the twisting angle of the Rf group reflecting the Rf length (Table 1). When the theoretical twisting angle is $90^{\circ}$ or larger, they are expected to aggregate spontaneously. ${ }^{4}$ When referring to

Table 1, below $19^{\circ} \mathrm{C}, \mathrm{MA}-\mathrm{Rf} n$ is spontaneously aggregated for $n=7$ and 9 (marked by bold in Table 1); whereas only MA-Rf9 should be aggregated above $19{ }^{\circ} \mathrm{C}$. This implies that only MA-Rf7 would exhibit an apparent change in both $\pi-A$ and $\Delta V-A$ isotherms when the measurement temperature is changed from $15{ }^{\circ} \mathrm{C}$ to $25^{\circ} \mathrm{C}$. 
1 that dehydration happens in this region because $\pi$ begins to increase after the suppress

16 finishes in an exchanging fashion. In this manner, only the MA-Rf7 monolayer exhibits

17 different aggregate properties in both $\pi-A$ and $\Delta V-A$ isotherms as predicted by the SDA theory.
Table 1 Theoretical twisting angle of both ends of the $\mathbf{C F}_{2}$ groups in an $\mathbf{R f}$ group as a function of the number of $\mathrm{CF}_{2}$ groups (Rf length) in Phases II and IV.

\begin{tabular}{ccc}
\hline \multirow{2}{*}{ No. of $\mathrm{CF}_{2}$} & \multicolumn{2}{c}{ Twisting angle $/{ }^{\circ}$} \\
\cline { 2 - 3 } & Phase II $\left(<19^{\circ} \mathrm{C}\right)$ & Phase IV $\left(>19^{\circ} \mathrm{C}\right)$ \\
\hline 3 & Un-twisted & Un-twisted \\
5 & 60 & 51 \\
7 & $\mathbf{9 0}$ & 77 \\
9 & $\mathbf{1 2 0}$ & $\mathbf{1 0 3}$ \\
\hline
\end{tabular}

Figure 5 presents the $\pi-A$ and $\Delta V-A$ isotherms of MA-Rfn monolayers on water at $25^{\circ} \mathrm{C} . \Delta V-A$ isotherms (solid lines) are particularly sensitive to the Rf length and temperature. As theoretically predicted on the SDA theory, only the $\Delta V-A$ isotherm of MA-Rf7 (orange curve) is significantly changed from that in Figure 2a; whereas the rest curves are kept almost unchanged. The potential decrease below the surface area of $0.9 \mathrm{~nm}^{2}$ molecule ${ }^{-1}$ in Figure 5 is more apparent than that of Figure 2a. The early decrease implies that the molecules at $25{ }^{\circ} \mathrm{C}$ are more homogeneously spread on water than $15{ }^{\circ} \mathrm{C}$. The decrease is suppressed at $0.7 \mathrm{~nm}^{2}$ molecule ${ }^{-1}$ or smaller, which indicates 


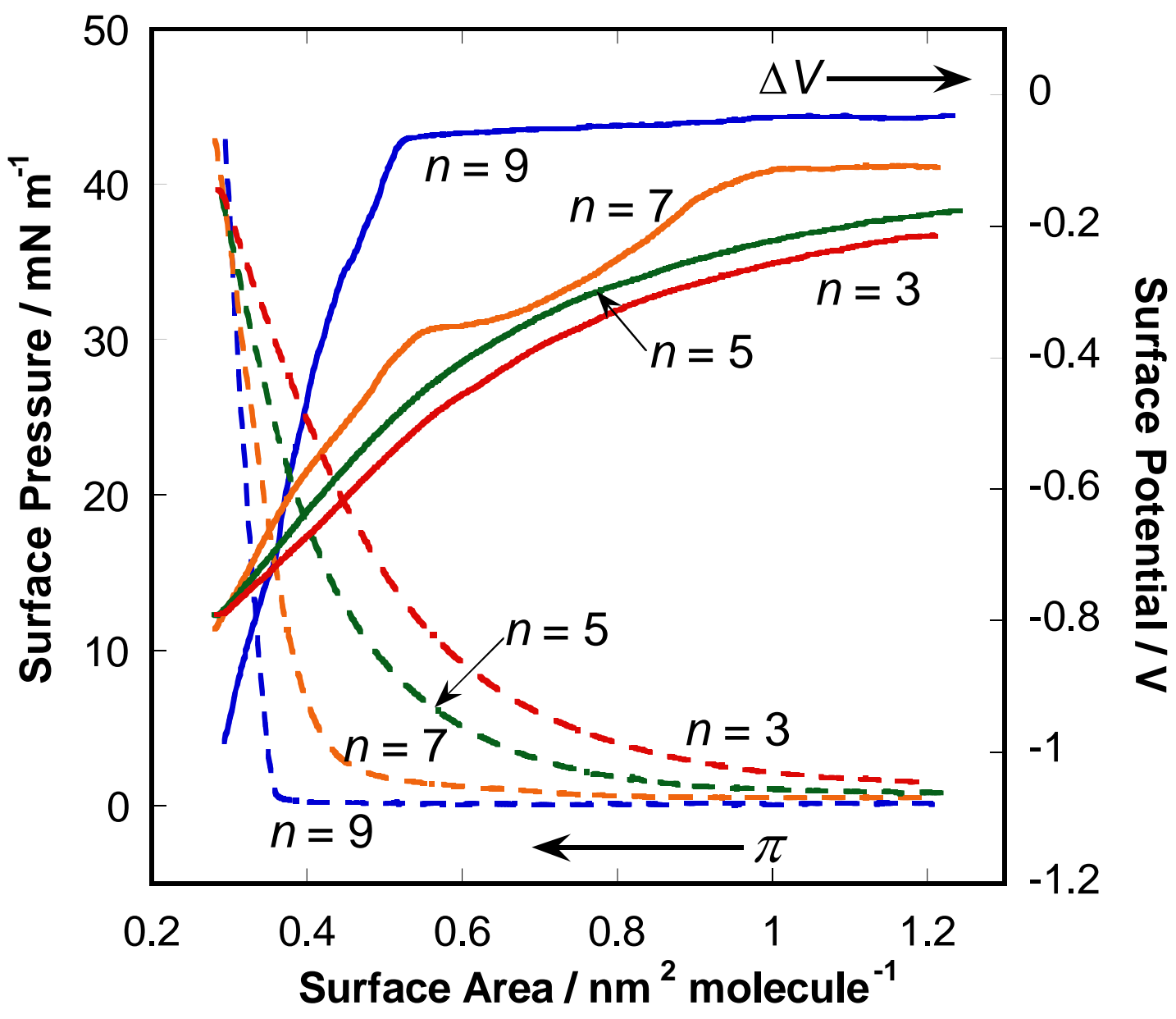

3 Figure 5. The $\pi-A$ (dashed line) and $\Delta V-A$ (solid line) isotherms of Langmuir monolayers of MA-Rf3 (red), MA-Rf5 (green), MA-Rf7 (orange) and MA-Rf9 (blue) at $25^{\circ} \mathrm{C}$ (phase IV).

6

8 check the homogeneity of the monolayer. When a monolayer of homogeneously spread

9 molecules is compressed, the $\Delta V-A$ measurements shows a good reproducibility;

10 whereas a monolayer of island domains shows a poor reproducibility. ${ }^{31}$ Figure 6 shows 
1 three traces for each $\pi-A$ and $\Delta V-A$ isotherm of MA-Rf3 and MA-Rf7. As shown in

2 Figure $6 \mathrm{a}, \mathrm{MA}-\mathrm{Rf} 3$ at $15{ }^{\circ} \mathrm{C}$ shows an extremely high reproducibility, and the same

3 trend is also found at $25^{\circ} \mathrm{C}$ (data not shown), which is consistent with a fact that a short

4 Rf compound does not shown an apparent aggregation. ${ }^{4}$ On the other hand, MA-Rf7

5 shows a good reproducibility at $25^{\circ} \mathrm{C}$; whereas the reproducibility is lost at $15{ }^{\circ} \mathrm{C}$ in the

6 area range of $0.4 \sim 0.6 \mathrm{~nm}^{2}$ molecule ${ }^{-1}$, which straightforwardly implies that the

7 molecules are aggregated to generate island domains at $15{ }^{\circ} \mathrm{C}$, while they are spread

8 homogeneously at $25{ }^{\circ} \mathrm{C}$. The results are perfectly consistent with the theoretical

9 prediction on the SDA theory (Table 1). 

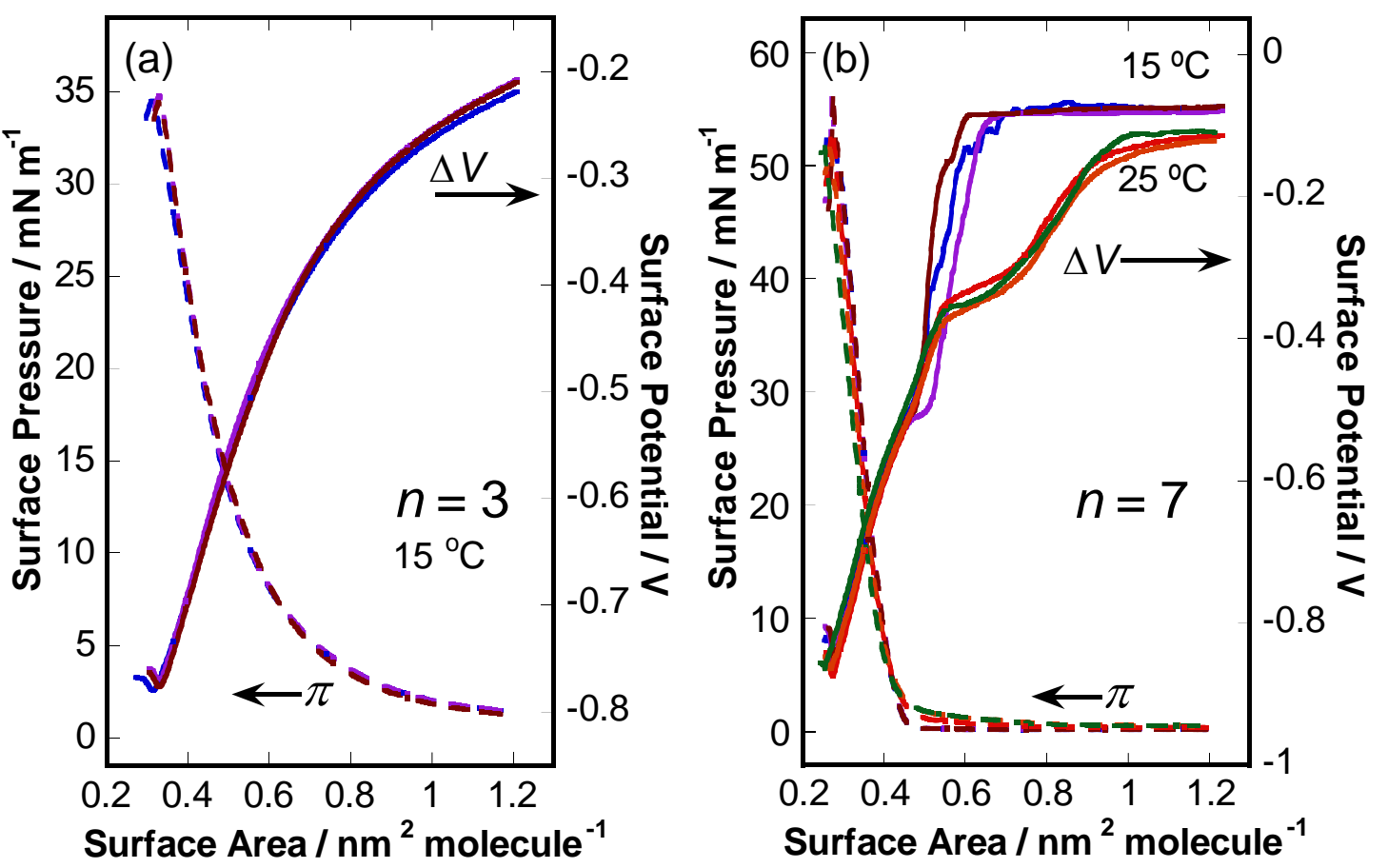

Figure 6. The $\pi-A$ (dashed lines) and $\Delta V$-A (solid lines) isotherms of the monolayers of (a) MA-Rf3 and (b) MA-Rf7. Three repeatedly measured results are overlaid for checking the reproducibility. Only the results at $15{ }^{\circ} \mathrm{C}$ are presented for better visibility in (a), since no difference was found for both $15^{\circ} \mathrm{C}$ and $25^{\circ} \mathrm{C}$.

The reader may be interested in the results of MA-Rf9 at $25^{\circ} \mathrm{C}$, since the

suppression of the potential decrease during the monolayer compression is not obvious

(Figure 5) when comparing to the results at $15{ }^{\circ} \mathrm{C}$. On closer inspection, however, the

11 suppression gradually appears along the potential decrease. Since the reproducibility of

12 this isotherm was high, the hydrated water should smoothly and gradually be removed

13 at $25{ }^{\circ} \mathrm{C}$ on the compression probably due to a high molecular mobility at a higher temperature. 
1 Conclusion: Through the comparative study of the surface potential $(\Delta V)$-surface area

2 (A) isotherm of a monolayer of a carboxylic acid involving a normal alkyl and a

3 perfluoro alkyl (Rf) group with a different length, the dehydration process is found to be

4 a key to understand the isotherms, which is a progress to the conventional discussion on

5 only the molecular density and orientation changes [15]. The 'dehydration' effect would

6 be useful for understanding the properties of a Langmuir monolayer of a wide range of

7 compounds, not only for Rf compounds. In particular for an Rf containing diblock

8 compound, the dehydration process is apparently visible in the isotherm, which greatly

9 helps us to discuss the surface pressure $(\pi)-A$ isotherm, too. As a result, the $\Delta V$

10 measurements have proved to be powerful to discriminate whether the compound has a

11 spontaneous molecular aggregation property or not. In addition, a lying Rf-R diblock

12 molecule has been revealed to have a "dipole-interactive property" on the Rf group. A

13 "single" Rf group is interacted with the water surface, but it is not dissolved in the bulky

14 water. In other words, the water surface has proved to have a unique chemical field to be

15 interacted with an Rf group. Regardless, a further molecular orientation study is

16 expected to fully confirm the discussion in this study. To do that, we are planning an

17 additional study using the polarization modulation-infrared reflection- absorption 
1 monolayer on the water surface in situ $[23,24]$.

2 


\section{ACKNOWLEDGMENT}

2 This work was financially supported by Grant-in-Aid for Young Scientists (B) [No.

3 26810075(TS)] and Grant-in-Aid for Scientific Research (A) (No. 15H02185 (TH))

4 from Japan Society for the Promotion of Science, the Collaborative Research Program

5 of Institute for Chemical Research, Kyoto University (grant no. 2015-87 (MS)), and the

6 "Element Innovation" Project by the Ministry of Education, Culture, Science, Sports

7 and Technology of Japan (MEXT), for which the authors thanks are due.

\section{REFERENCES:}

9 1. Krafft, M. P.; Riess, J. G. Chemistry, Physical Chemistry, and Uses of Molecular

10 Fluorocarbon-Hydrocarbon Diblocks, Triblocks, and Related Compounds-Unique

11 "Apolar" Components for Self-Assembled Colloid and Interface Engineering.

12 Chem. Rev. 2009, 109, 1714-1792.

2. Minkin, V. I.; Osipov, O. A.; Zhdanov, Y. A. Dipole Moments in Organic Chemistry; Plenum Press: New York, 1970.

3. Huheey, J. E. Inorganic Chemistry; Harper\&Row: New York, 1978.

4. Hasegawa, T.; Shimoaka, T.; Shioya, N.; Morita, K.; Sonoyama, M.; Takagi, T.; 
1 Specific to Perfluoroalkyl Compounds. ChemPlusChem 2014, 79, 1421-1425.

2 5. London, F. The General Theory of Molecular Forces. Trans. Faraday Soc. 1937, 8-

26.

6. Bunn, C. W.; Howells, E. R. Structures of Molecules and Crystals of Fluorocarbons. Nature 1954, 174.

6 7. Ute, K.; Kinoshita, R.; Matsui, K.; Miyatake, N.; Hatada, K. Conformational Asymmetry of a Linear Perfluoroalkyl Chain in Solution. Dynamic Fluorine-19 NMR Spectroscopy of the Perfluoro-n-Alkanes Carrying a Chiral End-Group as a Probe of Magnetic Nonequivalence. Chemistry Letters 1992, 1337-1340.

8. Thomas, R. R. Material properties of fluoropolymers and perfluoroalkyl-based polymers (Chapter 2), Fluoropolymers, vol. 2, Springer: Heidelberg, 1999.

9. Hasegawa, T. Understanding of the Intrinsic Difference between Normal- and Perfluoro-Alkyl Compounds toward Total Understanding of Material Properties. Chem. Phys. Lett. 2015, 627, 64-66.

10. Skrabania, K.; von Berlepsch, H.; Böttcher, C.; Laschewsky, Synthesis of Ternary, Hydrophilic-Lipophilic-Fluorophilic Block Copolymers by Consecutive RAFT Polymerizations and Their Self-Assembly into Multicompartment Micelles A. Macromol. 2010, 43, 271-281. 
1 11. Wakai, C.; Shimoaka, T.; Hasegawa, T. Characterization of Adsorbed Molecular Water on the Surface of a Stretched Polytetrafluoroethylene Tape Analyzed by ${ }^{1} \mathrm{H}$ NMR. J. Phys. Chem. B 2016, 120, 2538-2543.

12. Goto, T.; Ikehata, A.; Morisawa, Y.; Ozaki, Y. Surface Effect of Alumina on the First Electronic Transition of Liquid Water Studied by Far-Ultraviolet Spectroscopy. J. Phys. Chem. Lett. 2015, 6, 1022-1026.

13. Ariga, K.; Yamauchi Y.; Mori, T.; Hill J. P. 25th Anniversary Article: What Can Be Done with the Langmuir-Blodgett Method? Recent Developments and its Critical Role in Materials Science, Adv. Mater. 2013, 25, 6477-6512.

14. Albrecht, O.; Matsuda, H.; Eguchi, K.; Nakagiri, T. The Dissolution of Myristic Acid Monolayers in Water. Thin Solid Films 1999, 338, 252-264.

15. Gains, G. L., Jr. Insoluble monolayers at liquid-gas interface; John Wiley \& Sons, Inc: New York, 1966.

16. Clark, E. S. The Molecular Conformations of Polytetrafluoroethylene: Forms II and IV. Polymer 1999, 40, 4659-4665.

17. Yamins, H. G.; Zisman, W. A New Method of Studying the Electrical Properties of Monomolecular Films on Liquids. J. Chem. Phys. 1933, 1, 656.

18. Kang, Y. S.; Lee, D. K.; Kim, Y. S. A Study on Temperature Dependency and in 
Situ Observation of Domain Structure in Langmuir Layers of Stearic Acid $/ \gamma-\mathrm{Fe}_{2} \mathrm{O}_{3}$

Nanoparticle Complex at the Air/water Interface. Synthetic Metals 2001, 117, 165167.

19. MacRitchie, F. Monolayers (Chapter 7), Chemistry at Interfaces, Academic Press: San Diego, 1990.

20. Kaganer, V. M.; Möhwald, H.; Dutta, P. Structure and Phase Transitions in Langmuir Monolayers. Rev. Mod. Phys. 1999, 71, 3, 779-819.

21. Ouyang, B.; Howard, B. J. High-Resolution Microwave Spectroscopic and Ab Initio Studies of Propanoic Acid and Its Hydrates. J. Phys. Chem. A 2008, 112, 8208-8214.

22. Tanaka, H.; Hayashi, K.; Akatsuka, T.; Toyama, J.; Noda, K.; Kida, T.; Ogoma, Y.; Fujii, T.; Kondo, Y. Morphology of a Cytochrome c-Adsorbed Stearic Acid Monolayer on Brewster Angle Microscopy. J. Biochem. 1995, 117, 1151-1155.

23. Muro, M.; Itoh, Y.; Hasegawa, T. A Conformation and Orientation Model of the Carboxylic Group of Fatty Acids Dependent on Chain Length in a Langmuir Monolayer Film Studied by Polarization-Modulation Infrared Reflection Absorption Spectroscopy. J. Phys. Chem. B 2010, 114, 11496-11501.

24. Calvez, E.; Blaudez, D.; Buffeteau, T.; Desbat, B. Effect of Cations on the 
Dissociation of Arachidic Acid Monolayers on Water Studied by

Polarization-Modulated Infrared Reflection-Absorption Spectroscopy. Langmuir 2001, 17, 670-674.

25. Maaloum, M.; Muller, P.; Krafft, M. P. Monodisperse Surface Micelles of Nonpolar Amphiphiles in Langmuir Monolayers. Angew. Chem. 2002, 41, 4331-4334.

26. Lux, C.; Gallani, J.; Waton, G.; Krafft, M. P. Compression of Self Assembled Nano Objects: 2D/3D Transitions in Films of (Perfluoroalkyl) Alkanes Persistence of an Organized Array of Surface Micelles. Chem. - Eur. J. 2010, 16, 7186-7198.

27. Broniatowski, M.; Dynarowicz-Łątka, P. Semifluorinated Alcohols in Langmuir monolayers-A Comparative Study. J. Colloid Interface Sci. 2006,301, 315-322.

28. Hasegawa, T.; Shimoaka, T.; Tanaka, Y.; Shioya, N.; Morita, K.; Sonoyama, M.; Amii, H.; Takagi, T.; Kanamori, T. An Origin of Complicated Infrared Spectra of Perfluoroalkyl Compounds Involving a Normal Alkyl Group. Chem. Lett. 2015, 44, $834-836$.

29. Kato, T; Kameyama, M; Ehara, M; Iimura, K. Monodisperse Two-Dimensional Nanometer Size Clusters of Partially Fluorinated Long-Chain Acids. Langmuir 1998, 14, 1786-1798.

30. Bernett, M. K.; Zisman, W. A. The Behavior of Monolayers of Progressively 
Fluorinated Fatty Acids Adsorbed on Water. J. Phys. Chem. 1963, 67, 1534-1540.

2 31. Harkins, W. D.; Fischer, E. K. Contact Potentials and the Effects of Unimolecular

3

4 Films on Surface Potentials. I. Films of Acids and Alcohols. J. Chem. Phys. 1933, 1, $852-862$. 